\title{
Stability Analysis of Small Satellite Formation Flying and Reconfiguration Missions in Deep Space
}

\author{
Saptarshi Bandyopadhyay, Chakravarthini M. Saaj, and Bijnan Bandyopadhyay, Member, IEEE
}

\begin{abstract}
Close-proximity (10-150 m) formation flying using low cost, small satellites is an emerging field. In such missions, control of satellite formations is a challenging problem and requires robust on-board control systems. This paper describes a modified approach to designing Sliding Mode Control (SMC) for satellite formation and reconfiguration missions, in deep space with external disturbances. Based on this dynamic model, a new approach for implementing path planning of satellites using Artificial Potential Field (APF) method is presented in this paper. This paper discusses stability of the sliding surfaces designed using gradient of the potential function for the closed loop system. The stability analysis is demonstrated by presenting a scenario in which six satellites aggregates to form an octahedron formation and subsequently reconfigure to a hexagon formation. This paper thus presents further progress in the state of-the-art of path planning and control for the framework of satellite formation and reconfiguration missions.
\end{abstract}

\section{INTRODUCTION}

A formation of small satellites, operating in a cluster or predefined geometry, can accomplish the task of a single, conventional large satellite [1]. The risk of inter-satellite collision is significant, when small satellites are required to fly in close formations ( $<50 \mathrm{~m}$ inter-satellite separation). Therefore, an intelligent path planning technique is required for collision free navigation. An efficient on-board controller also plays a vital role in extending the life-time of the mission through minimising the fuel consumption.

Swarming is a widely observed phenomenon in nature [2]. In [3-5], the biologists have studied the effect of cohesion for a family of attraction/repulsion functions and provide good background and review of the swarm modelling concepts.

The major focus of this paper is on improving the performance of the controller for collision free navigation of the satellites for aggregation and formation flying. A robust control algorithm using state feedback SMC is proposed for formation flying and reconfiguration missions. Intelligent path planning algorithm is designed using the APF method [6]. This paper presents how satellites in a formation can aggregate towards a goal position, similar to biological swarms and then takes up positions to form a predefined formation. Based on the knowledge of current position, the APF method will optimise the trajectory to generate the next

Manuscript received September 14, 2011. This two year project was supported by the Royal Society, UK, through the International Joint Project, which commenced in April 2009.

Saptarshi Bandyopadhyay was with the Department of Aerospace Engineering, Indian Institute of Technology Bombay, Mumbai, India. (email: saptarshi.bandyopadhyay@gmail.com).

Chakravarthini M. Saaj is with the Surrey Space Centre, University of Surrey, Guildford, United Kingdom. (corresponding author, phone: +44.1483682225; fax: +44.1483689503; e-mail: c.saaj@surrey.ac.uk).

Bijnan Bandyopadhyay is with the Department of Systems \& Control Engineering, Indian Institute of Technology Bombay, Mumbai, India. (email: bijnan@ee.iitb.ac.in) desired formation that will be achieved using SMC. Since SMC guarantees robust performance, the impact of external perturbations like solar wind and internal perturbations like change in mass due to fuel consumption will have negligible impact on the performance of the satellite [7].

This paper presents how satellites, whose dynamics are governed by the Deep Space Environment, can aggregate towards a goal position to form a predefined formation. In Section II, the background results on APF and dynamics in deep space are presented. Based on the knowledge of current position of the satellite, the APF method will create the trajectory to generate the desired formation, which will be achieved using the sliding mode controller discussed in Section III. Further, the stability of the sliding motion along the sliding surfaces is discussed in Section IV. Simulation results for a formation and reconfiguration scenario with six satellites are presented in Section V. Conclusions and future scope of this research is presented in Section VI.

\section{BACKGROUND RESULTS}

\section{A. Path Planning Using Artificial Potential Field}

Let the formation consist of $n$ individual satellites, and the position of the $i^{\text {th }}$ satellite is described by $\boldsymbol{p}_{\boldsymbol{i}}$. The motion of each satellite in the formation is governed by the equation:

$$
\dot{\boldsymbol{p}}_{\boldsymbol{i}}=-\sum_{j=1, j \neq i}^{n} g\left(\boldsymbol{p}_{\boldsymbol{i}}-\boldsymbol{p}_{\boldsymbol{j}}\right), \quad i=1, \ldots, n,
$$

where $g($.$) is an artificial potential function [9]. It is an odd$ function which represents the net attraction and repulsion between the satellites. It is needed for aggregation that the attraction term dominates on large distances and for avoiding collisions that the repulsion term dominates on short distances. The potential function that is considered in this study is:

$$
g\left(\boldsymbol{p}_{\boldsymbol{i}}-\boldsymbol{p}_{\boldsymbol{j}}\right)=\left(\boldsymbol{p}_{\boldsymbol{i}}-\boldsymbol{p}_{\boldsymbol{j}}\right) \cdot\left(a_{i j}-b_{i j} \exp \left(\frac{-\left\|\boldsymbol{p}_{\boldsymbol{i}}-\boldsymbol{p}_{\boldsymbol{j}}\right\|^{2}}{c_{i j}}\right)\right)
$$

where $a_{i j}, b_{i j}$, and $c_{i j}$ are positive constants such that $b_{i j}>a_{i j}$. The term $a_{i j}$ represents the attraction that dominates for large distances, whereas the term $b_{i j} \exp \left(-\left\|\boldsymbol{p}_{\boldsymbol{i}}-\boldsymbol{p}_{j}\right\|^{2} / c_{i j}\right)$ represents the repulsion and dominates for small distances. There is a distance $\delta_{i j}=$ $\sqrt{c_{i j} \ln \left(b_{i j} / a_{i j}\right)}$ at which the attraction and repulsion balance [8]. The main drawback with $g($.$) is that the maximum$ repulsion is bounded for infinitesimally small distances. In practice, unbounded repulsion may be needed to avoid collisions below a threshold distance. Another drawback is that it has an infinite range, which is inconsistent with biological systems. 


\section{B. Dynamics of Formation Flying Satellites in Deep Space Enviornment}

The ability to accurately model the dynamic behaviour of separated spacecraft formations is critical to the success of the mission. It is assumed that the satellite is not affected by any significant gravitational forces when it is in Deep Space Environment. Hence a simple double integrator model is used to represent the dynamics of the swarm agents.

$$
\ddot{\boldsymbol{p}}_{\boldsymbol{i}}=\frac{\boldsymbol{F}_{\boldsymbol{i}}+\boldsymbol{D}_{\boldsymbol{i}}}{m_{i}}
$$

where $\boldsymbol{F}_{\boldsymbol{i}}$ is the actuator forces and $\boldsymbol{D}_{\boldsymbol{i}}$ are the disturbance forces acting on the $i^{\text {th }}$ satellites.

\section{Design OF SLIDING Mode CONTROLLER}

In this section, the APF based path planning and SMC based control algorithms are implemented for a satellite formation in Deep Space Environment. When the satellite is in orbit, it experiences disturbances due to the oblateness of Earth, aerodynamic drag, solar drag, etc which is accounted using the disturbance term. The open loop dynamic equation for the $i^{\text {th }}$ satellite in the orbit frame is given by equation (3). The APF equation (1) gives the switching hyperplane $\boldsymbol{s}_{\boldsymbol{i}} \in \boldsymbol{R}^{\mathbf{3}}$ for the $i^{\text {th }}$ satellite and is given by:

$$
\begin{aligned}
& \boldsymbol{s}_{\boldsymbol{i}}=\dot{\boldsymbol{p}}_{\boldsymbol{i}}+\sum_{j=1, j \neq i}^{n} g\left(\boldsymbol{p}_{\boldsymbol{i}}-\boldsymbol{p}_{\boldsymbol{j}}\right), \quad i=1, \ldots, n, \quad \text { (4) } \begin{array}{l}
\text { controller is used. Also, } \epsilon_{i, k}>\max \left(\left\|D_{k i}\right\|\right) \text { for } k=x, y, z . \\
\text { Differentiating }(5) \text { and equating it to }(6) \text { gives: }
\end{array} \\
&-\gamma_{i} \boldsymbol{s}_{\boldsymbol{i}}-\left[\begin{array}{ccc}
\epsilon_{i, x} & 0 & 0 \\
0 & \epsilon_{i, y} & 0 \\
0 & 0 & \epsilon_{i, z}
\end{array}\right] * \boldsymbol{f} \mathbf{s a t}\left(\boldsymbol{s}_{\boldsymbol{i}}, \boldsymbol{\phi}\right) \\
&=\ddot{\boldsymbol{p}}_{\boldsymbol{i}}+\sum_{j=1, j \neq i}^{n}\left(\left(\dot{\boldsymbol{p}}_{\boldsymbol{i}}-\dot{\boldsymbol{p}}_{\boldsymbol{j}}\right) \cdot\left(a_{i j}-b_{i j} \exp \left(\frac{-\left\|\boldsymbol{p}_{\boldsymbol{i}}-\boldsymbol{p}_{\boldsymbol{j}}\right\|^{2}}{c_{i j}}\right)\right)+\frac{b_{i j}}{c_{i j}}\left(\boldsymbol{p}_{\boldsymbol{i}}-\boldsymbol{p}_{\boldsymbol{j}}\right) \cdot\left(\exp \left(\frac{-\left\|\boldsymbol{p}_{\boldsymbol{i}}-\boldsymbol{p}_{\boldsymbol{j}}\right\|^{2}}{c_{i j}}\right)\right) \cdot\left(\frac{d}{d t}\left\|\boldsymbol{p}_{\boldsymbol{i}}-\boldsymbol{p}_{\boldsymbol{j}}\right\|^{2}\right)\right)
\end{aligned}
$$

Substituting (2) into (4) gives:

$$
\boldsymbol{s}_{\boldsymbol{i}}=\dot{\boldsymbol{p}}_{\boldsymbol{i}}+\sum_{j=1, j \neq i}^{n}\left(\boldsymbol{p}_{\boldsymbol{i}}-\boldsymbol{p}_{j}\right) \cdot\left(a_{i j}-b_{i j} \exp \left(\frac{-\left\|\boldsymbol{p}_{\boldsymbol{i}}-\boldsymbol{p}_{j}\right\|^{2}}{c_{i j}}\right)\right)
$$

where $a_{i j}, b_{i j}$, and $c_{i j}$ are the tuning parameters for APF. If $\delta_{i j}$ is the final distance to be maintained between the $i^{\text {th }}$ and $j^{\text {th }}$ satellites, then $a_{i j}=b_{i j} \exp \left(-\delta_{i j}^{2} / c_{i j}\right)$.

In SMC, the sliding mode should start in finite time. The reaching condition $\boldsymbol{s} \dot{\boldsymbol{s}} \leq-\eta\|\boldsymbol{s}\|$, where $\eta$ is some positive number; will ensure the sliding mode is reached in finite time. This is ensured by the reaching law. Applying the constant plus proportional rate reaching law given in [10], for the $i^{\text {th }}$ satellite in Deep Space Environment gives:

$$
\begin{gathered}
\dot{\boldsymbol{s}}_{\boldsymbol{i}}=-\gamma_{i} \boldsymbol{s}_{\boldsymbol{i}}-\left[\begin{array}{ccc}
\epsilon_{i, x} & 0 & 0 \\
0 & \epsilon_{i, y} & 0 \\
0 & 0 & \epsilon_{i, z}
\end{array}\right] * \mathbf{f} \operatorname{sat}\left(\boldsymbol{s}_{i}, \phi\right) \\
\boldsymbol{s}_{\boldsymbol{i}} \in \boldsymbol{R}^{3 x 1}, \mathbf{f} \operatorname{sat}\left(\boldsymbol{s}_{i}, \phi\right)=\left[\operatorname{sat}\left(\frac{s_{i x}}{\phi}\right) \quad \operatorname{sat}\left(\frac{s_{i y}}{\phi}\right) \quad \operatorname{sat}\left(\frac{s_{i z}}{\phi}\right)\right]
\end{gathered}
$$

where $\gamma_{i}, \epsilon_{i, x}, \epsilon_{i, y}$, and $\epsilon_{i, z}$ are the tuning parameters and $\phi$ is the boundary layer around the sliding surface, within which a proportional based and not a switching based

Substituting for $\ddot{\boldsymbol{p}}_{\boldsymbol{i}}$ from (3) into (7), gives the control input:

$$
\begin{aligned}
\frac{\boldsymbol{F}_{\boldsymbol{i}}}{m_{i}}= & -\frac{\boldsymbol{D}_{\boldsymbol{i}}}{m_{i}}-\gamma_{i} \boldsymbol{s}_{\boldsymbol{i}}-\left[\begin{array}{ccc}
\epsilon_{i, x} & 0 & 0 \\
0 & \epsilon_{i, y} & 0 \\
0 & 0 & \epsilon_{i, z}
\end{array}\right] * \boldsymbol{f} \operatorname{sat}\left(\boldsymbol{s}_{\boldsymbol{i}}, \phi\right) \\
& -\sum_{j=1, j \neq i}^{n}\left(\left(\dot{\boldsymbol{p}}_{\boldsymbol{i}}-\dot{\boldsymbol{p}}_{j}\right) \cdot\left(a_{i j}-b_{i j} \exp \left(\frac{-\left\|\boldsymbol{p}_{\boldsymbol{i}}-\boldsymbol{p}_{\boldsymbol{j}}\right\|^{2}}{c_{i j}}\right)\right)+\frac{b_{i j}}{c_{i j}} \cdot\left(\boldsymbol{p}_{\boldsymbol{i}}-\boldsymbol{p}_{j}\right) \cdot\left(\exp \left(\frac{-\left\|\boldsymbol{p}_{\boldsymbol{i}}-\boldsymbol{p}_{j}\right\|^{2}}{c_{i j}}\right)\right) \cdot\left(\frac{d}{d t}\left\|\boldsymbol{p}_{\boldsymbol{i}}-\boldsymbol{p}_{j}\right\|^{2}\right)\right)
\end{aligned}
$$

The switching function will negate the influence of the external disturbance, and hence the closed loop system is robust. The closed loop dynamics is obtained by substituting the control input from (8) into the original system (3) as given below:

$$
\begin{aligned}
\ddot{\boldsymbol{p}}_{\boldsymbol{i}}= & -\gamma_{i} \boldsymbol{s}_{\boldsymbol{i}}-\left[\begin{array}{ccc}
\epsilon_{i, x} & 0 & 0 \\
0 & \epsilon_{i, y} & 0 \\
0 & 0 & \epsilon_{i, z}
\end{array}\right] * \mathbf{f} \mathbf{s a t}\left(\boldsymbol{s}_{\boldsymbol{i}}, \boldsymbol{\phi}\right) \\
& -\sum_{j=1, j \neq i}^{n}\left(\left(\dot{\boldsymbol{p}}_{\boldsymbol{i}}-\dot{\boldsymbol{p}}_{\boldsymbol{j}}\right) \cdot\left(a_{i j}-b_{i j} \exp \left(\frac{-\left\|\boldsymbol{p}_{\boldsymbol{i}}-\boldsymbol{p}_{j}\right\|^{2}}{c_{i j}}\right)\right)+\frac{b_{i j}}{c_{i j}}\left(\boldsymbol{p}_{\boldsymbol{i}}-\boldsymbol{p}_{\boldsymbol{j}}\right) \cdot\left(\exp \left(\frac{-\left\|\boldsymbol{p}_{\boldsymbol{i}}-\boldsymbol{p}_{\boldsymbol{j}}\right\|^{2}}{c_{i j}}\right)\right) \cdot\left(\frac{d}{d t}\left\|\boldsymbol{p}_{\boldsymbol{i}}-\boldsymbol{p}_{j}\right\|^{2}\right)\right)
\end{aligned}
$$

In ideal condition when $\boldsymbol{s}_{\boldsymbol{i}}=0$, the closed loop dynamics equation is independent of the original system parameters and the system will be robust when it is sliding on the sliding surface. SMC rejects the matched uncertainty or external disturbance and the sliding motion is governed by the sliding surface parameters. For good performance, it is observed that $c_{i j}$ is of the same order as $\delta_{i j}^{2}$. Moreover, the value of $b_{i j}$ should be chosen to avoid actuator saturation.

\section{STABILITY OF THE FORMATION}

The stability of the formation can be ensured by following a similar approach as given for satellite formation flying missions in Planetary Orbital Environments [11]. Assuming $\boldsymbol{s}_{\boldsymbol{i}}=0$ in (5), and $\rho_{i j}=\left\|\boldsymbol{p}_{\boldsymbol{i}}-\boldsymbol{p}_{j}\right\|$ is the distance between the $i^{t h}$ and $j^{\text {th }}$ satellite; the velocity of the $i^{t h}$ satellite is:

$\dot{\boldsymbol{p}}_{\boldsymbol{i}}=-\sum_{j=1, j \neq i}^{n}\left(\boldsymbol{p}_{\boldsymbol{i}}-\boldsymbol{p}_{\boldsymbol{j}}\right)\left(a_{i j}-b_{i j} \exp \left(\frac{-\rho_{i j}^{2}}{c_{i j}}\right)\right), i=1, \ldots, n$, 
Now expressing (10) in state space form by substituting, $\boldsymbol{p}_{\boldsymbol{i}}=\left[\begin{array}{lll}x_{i} & y_{i} & z_{i}\end{array}\right]^{T}$, gives:

$\left[\begin{array}{lllllllll}\dot{x_{1}} & \dot{y_{1}} & \dot{z_{1}} & \dot{x_{2}} & \dot{y_{2}} & \ldots & \dot{x_{n}} & \dot{y_{n}} & \dot{z}_{n}\end{array}\right]^{T}$

$=\mathbf{A}\left[\begin{array}{lllllllll}x_{1} & y_{1} & z_{1} & x_{2} & y_{2} & \ldots & x_{n} & y_{n} & z_{n}\end{array}\right]^{T}$

where $\mathbf{A} \in \boldsymbol{R}^{3 n \times 3 n}$ and the terms in $\mathbf{A}$ will include all the constant terms $\left(a_{i j}-b_{i j} \exp \left(-\rho_{i j}^{2} / c_{i j}\right)\right)$. The states should not asymptotically tend to zero; but converge towards the desired formation distance $\delta_{i j}$. Hence the following conditions must be satisfied:

(i) If $\forall \rho_{i j}>\delta_{i j}, i \neq j ; i=1, \ldots, n ; j=1, \ldots, n$; then the real part of all the eigenvalues of $\mathbf{A}$ will be negative, as all states should converge towards the origin of the state space.

(ii) If $\forall \rho_{i j}<\delta_{i j}, i \neq j ; i=1, \ldots, n$; $j=1, \ldots, n$; then the real part of some of the eigenvalues of $\mathbf{A}$ will be positive, as all states will diverge from the origin of the state space.

The variables $a_{i j}, b_{i j}$, and $c_{i j}$ should be so designed that the A matrix will obey the above rules. When the sliding surface is plotted, it is seen that the motion along the sliding surface is not stable for some regions. This property of the sliding surface is captured while designing the APF parameters, using this stability analysis. The equilibrium is reached when the sliding surface is stable and the intersatellite distance is as commanded. Plotting the intersection of the sliding surface shows the path that the satellites will take along the sliding surface to reach the stable equilibrium point.

The stability analysis for the simplified case of only two satellites can be proved using Lyapunov's Direct Method. Let us assume the position vectors of the two satellites are $\boldsymbol{p}_{\mathbf{1}}$ and $\boldsymbol{p}_{\mathbf{2}}$. The final desired inter-satellite separation is $\delta_{12}$. Let $\rho_{12}=\left\|\boldsymbol{p}_{\mathbf{1}}-\boldsymbol{p}_{\mathbf{2}}\right\|$ be the present inter-satellite separation between them. The velocity vectors for the two satellites are:

$\dot{\boldsymbol{p}_{1}}=-\left(\boldsymbol{p}_{\mathbf{1}}-\boldsymbol{p}_{\mathbf{2}}\right) C_{12}$, where $C_{12}=\left(a_{12}-b_{12} \exp \left(\frac{-\rho_{12}^{2}}{c_{12}}\right)\right)$

$\boldsymbol{p}_{2}=-\left(\boldsymbol{p}_{2}-\boldsymbol{p}_{1}\right) C_{21}$, where $C_{21}=\left(a_{21}-b_{21} \exp \left(\frac{-\rho_{12}^{2}}{c_{21}}\right)\right)$

$C_{12}$ and $C_{21}$ are so designed that $C_{12}, C_{21}>0, \forall \rho_{12}>\delta_{12}$, $C_{12}, C_{21}<0, \forall \rho_{12}<\delta_{12}$ and $C_{12}, C_{21}=0, \forall \rho_{12}=\delta_{12}$. Let the Lyapunov function $V=\rho_{12}^{2}$, which is positive definite. Differentiating with $V$ respecting to time gives:

$$
\begin{aligned}
\dot{V}=\frac{d}{d t} & \left(\rho_{12}^{2}\right)=\frac{d}{d t}\left(\left(\boldsymbol{p}_{\mathbf{1}}-\boldsymbol{p}_{2}\right)^{T}\left(\boldsymbol{p}_{\mathbf{1}}-\boldsymbol{p}_{\mathbf{2}}\right)\right) \\
& =2\left(\boldsymbol{p}_{\mathbf{1}}-\boldsymbol{p}_{\mathbf{2}}\right)\left(\boldsymbol{p}_{\mathbf{1}}-\boldsymbol{p}_{\mathbf{2}}\right)=-2 \rho_{12}^{2}\left(C_{12}+C_{21}\right)
\end{aligned}
$$

In equation (12), for $\dot{V}=0$, either $\rho_{12}=0$ or $\left(C_{12}+\right.$ $C 21=0$. But if $\rho 12$ is small $(<\delta 12)$, then $C 12+C 21<0$, therefore $\dot{V}>0$, hence system is unstable at this equilibrium point. For $\rho_{12}>\delta_{12}, \quad\left(C_{12}+C_{21}\right)>0$, hence $\dot{V}<0$. Moreover, $V>\delta_{12}^{2}$, hence $\rho_{12}$ is bounded for all time and stable. Hence system will tend to $\dot{V}=0$, i.e. $\left(C_{12}+C_{21}\right)=0$ and $\rho_{12}=\delta_{12}$. Hence the system tends to the stable equilibrium position.

\section{Simulation Results}

In this section, simulation results are presented for two scenarios, where six satellites, each weighing $10 \mathrm{~kg}$, are brought from a large initial distance to form an octahedron formation with inter-satellite separation of 50 meters and then the formation will be reconfigured to a hexagon formation with side of 50 meters.

\section{A. Scenario 1: Octahedron Formation}

First the APF parameters are found such that they satisfy the stability criterion. The corresponding $b$ and $c$ matrices used in this simulation are:

$$
\begin{gathered}
b=\left[\begin{array}{cccccc}
0 & 10^{-4} & 10^{-4} & 10^{-4} & 10^{-4} & 10^{-4} \\
10^{-4} & 0 & 10^{-4} & 10^{-4} & 10^{-4} & 10^{-4} \\
10^{-4} & 10^{-4} & 0 & 10^{-4} & 10^{-4} & 10^{-4} \\
10^{-4} & 10^{-4} & 10^{-4} & 0 & 10^{-4} & 10^{-4} \\
10^{-4} & 10^{-4} & 10^{-4} & 10^{-4} & 0 & 10^{-4} \\
10^{-4} & 10^{-4} & 10^{-4} & 10^{-4} & 10^{-4} & 0
\end{array}\right] \\
c=\left[\begin{array}{cccccc}
0 & 800 & 800 & 800 & 800 & 800 \\
800 & 0 & 1000 & 900 & 800 & 800 \\
900 & 1100 & 0 & 900 & 800 & 800 \\
1100 & 1100 & 800 & 0 & 800 & 800 \\
1100 & 1100 & 800 & 800 & 0 & 800 \\
1100 & 1100 & 800 & 800 & 800 & 0
\end{array}\right]
\end{gathered}
$$

The terms in the $a$ matrix are found using $a_{i j}=$ $b_{i j} \exp \left(-\delta_{i j}^{2} / c_{i j}\right)$, where $\delta_{i j}$ is 50 meters between all $i^{t h}$ and $j^{\text {th }}$ satellites. Fig. 1 shows the plot of the maximum eigenvalue of $\mathbf{A}$ matrix for these APF variables when the inter-satellite distance is varied from 1 to 2000 meters for the tetrahedron formation. As expected, for all inter-satellite distances less than 50 meters, the maximum eigenvalue of $\mathbf{A}$ is positive and for all inter-satellite distances more than 50 meters, the maximum eigenvalue of $\mathbf{A}$ is negative.

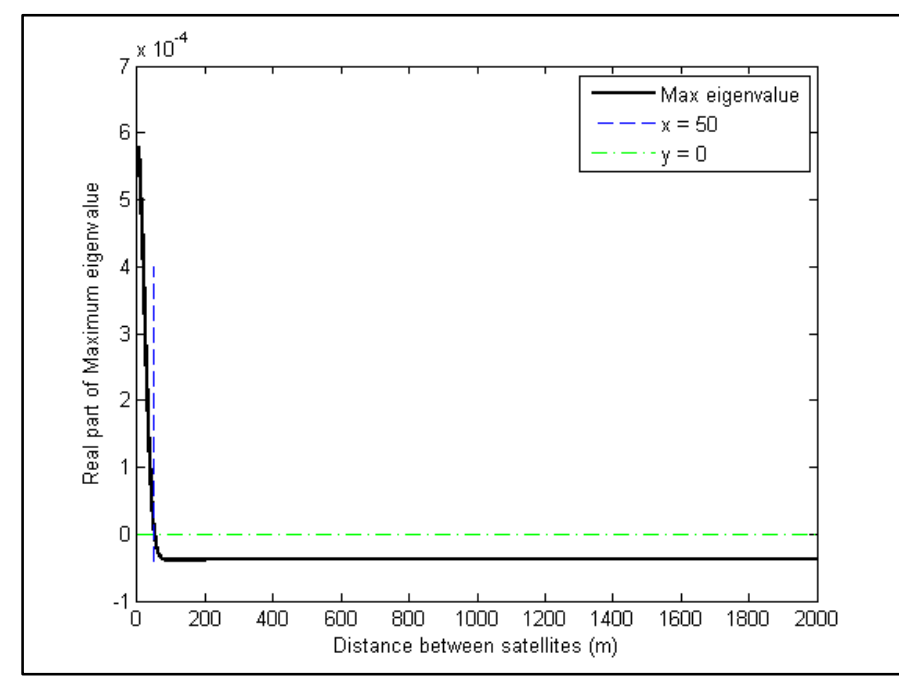

Figure 1: Octahedron Formation: Maximum Eigenvalue of the A Matrix

The six satellites that form the octahedron formation start from an initial separation distance of approximately $500 \mathrm{~m}$ $1 \mathrm{~km}$. The initial coordinates for the location of the satellites are randomly selected as follows: Sat1 (300, 0, 0), Sat2 (0, 50, 300), Sat3 (10, 300, 0), Sat4 (-10, -30, 0), Sat5 (0, 0, $100)$ and Sat6 $(-300,-320,-200)$. It is assumed that each of the satellites has three thrusters which produce thrust independently along the three axis. The maximum thrust that can be produced by each thruster is limited to $7.7 \mathrm{mN}$ per $\mathrm{kg}$, as given in [12]. The random external disturbance acting on the formation has a maximum bound of $\pm 10^{-4} \mathrm{~N}$. Some of the other parameters used for the simulation are $\gamma_{i j}=1.0, \epsilon_{i j}=$ 
$1.0 \forall i, j$ and $\phi=0.001$. The simulation was executed for $4 \times 10^{5}$ seconds, which is about 4.5 days.

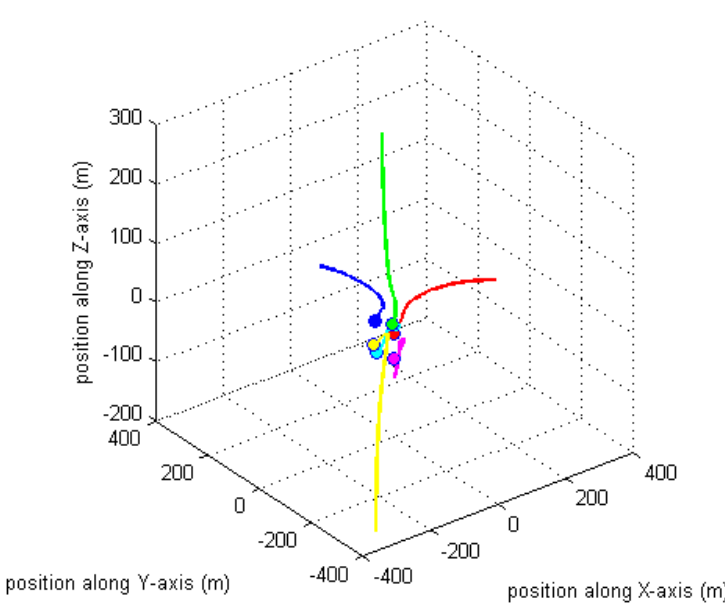

Figure 2: Octahedron Formation: Aggregation of Satellites

Table 1. Octahedron Formation: Final Inter-Satellite Separation

\begin{tabular}{|l|r|r|r|r|r|r|}
\hline & \multicolumn{1}{|c|}{ Sat 1 } & \multicolumn{1}{c|}{ Sat 2 } & \multicolumn{1}{l|}{ Sat 3 } & \multicolumn{1}{c|}{ Sat 4 } & \multicolumn{1}{|c|}{ Sat 5 } & \multicolumn{1}{c|}{ Sat 6 } \\
\hline Sat 1 & 0.0 & 47.6 & 52.0 & 52.4 & 54.9 & 72.3 \\
\hline Sat 2 & 47.6 & 0.0 & 51.0 & 69.9 & 55.0 & 52.7 \\
\hline Sat 3 & 52.0 & 51.0 & 0.0 & 50.5 & 78.7 & 53.8 \\
\hline Sat 4 & 52.4 & 69.9 & 50.5 & 0.0 & 54.1 & 48.3 \\
\hline Sat 5 & 54.9 & 55.0 & 78.7 & 54.1 & 0.0 & 53.0 \\
\hline Sat 6 & 72.3 & 52.7 & 53.8 & 48.3 & 53.0 & 0.0 \\
\hline
\end{tabular}

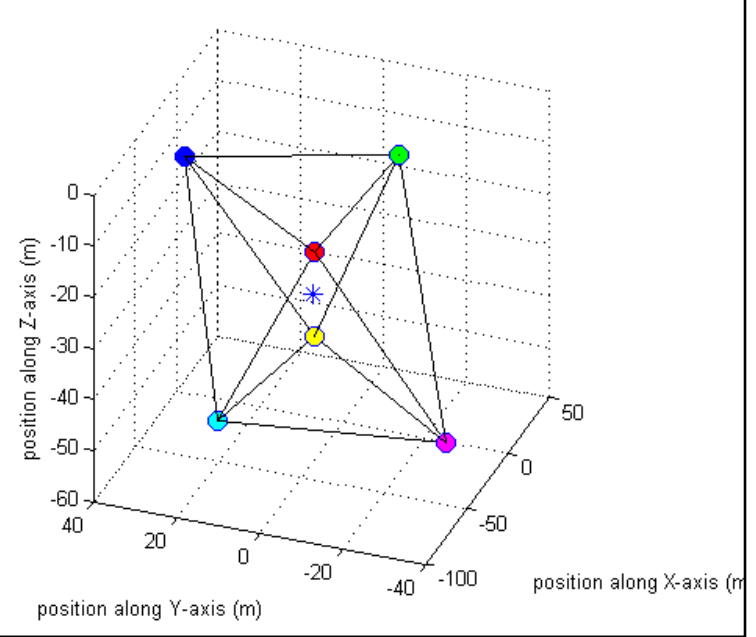

Figure 3: Octahedron Formation: Final Formation with the Centre of Mass

Fig. 2 shows the trajectory of the six satellites as they head towards the formation and then slowly drift in a particular direction. Fig. 3 shows the final position of the satellites in the octahedron formation. The final inter-satellite separations are shown in Table 1, which proves that the final inter-satellite distances are well within tolerable limits.

The formation is successfully achieved in $2 \times 10^{5}$ seconds as can be inferred from the inter-satellite distances plotted in Fig. 4(a). The simulation results show that the position of the Centre of Mass of the formation changes very little while the satellites are aggregating towards the formation. The relative velocity $\left(\dot{\boldsymbol{p}}_{\boldsymbol{i}}-\dot{\boldsymbol{p}}_{\boldsymbol{j}}, \forall i \neq j\right)$ between the satellites is shown in Fig. 4(b). Once the formation is achieved, the relative velocities are very low, i.e. in the range of $8-1.5 \times 10^{-5} \mathrm{~m} / \mathrm{s}$.

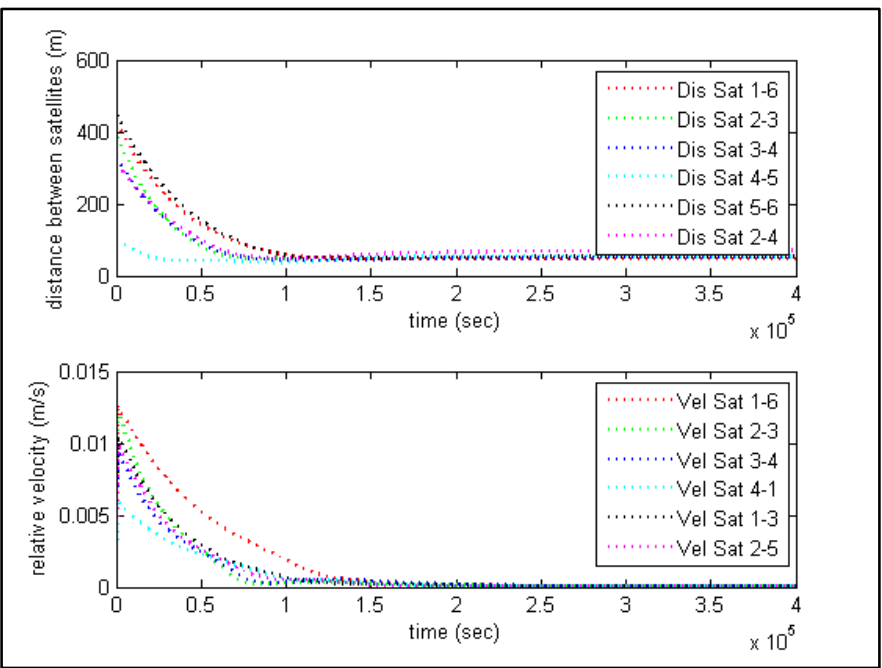

Figure 4: Octahedron Formation: (a) Distance between the satellites (b) Relative Velocities between satellites
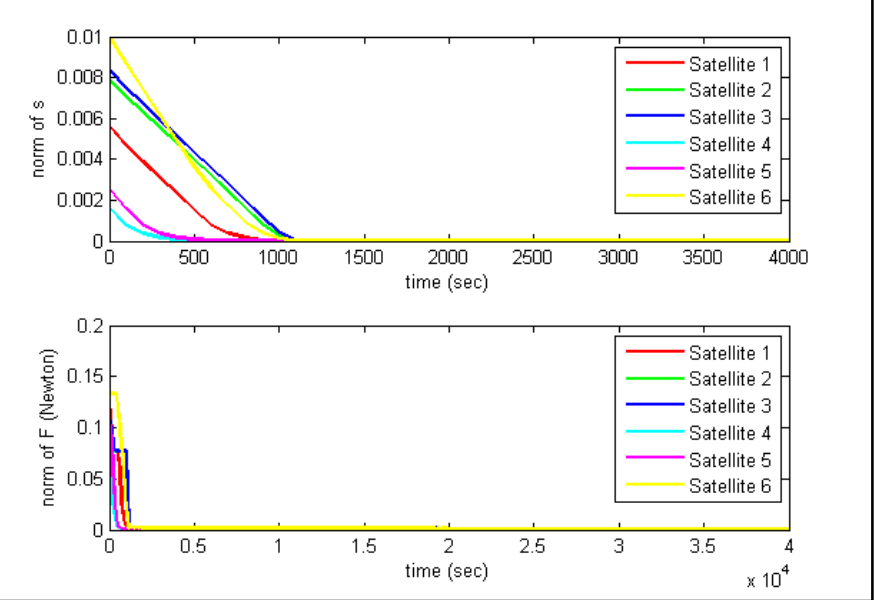

Figure 5: Octahedron Formation: (a) Norm of the sliding surface $s_{i}(b)$ Norm of the Force applied by each satellite

In Fig. 5(a), the norm of sliding surface is plotted, where $\left\|s_{i}\right\|=\sqrt{s_{i x}^{2}+s_{i y}^{2}+s_{i z}^{2}}$. The reaching phase is visible and the system states hits the sliding surface within approximately 2000 seconds. Thereafter the system continues in the sliding surface towards the formation. The thickness of the boundary layer is less than $10^{-4}$. Fig. 5(b) shows the plot of the total magnitude of the force required by the individual satellites for the formation to be achieved, where $\left\|F_{i}\right\|=$ $\sqrt{F_{i x}^{2}+F_{i y}^{2}+F_{i z}^{2}}$. The forces expended by the individual satellites are close to the minimum thrust requirements in [12]. 


\section{B. Scenario 2: Reconfiguration from Octahedron to Hexagonal Formation}

Next, the above formation was reconfigured to form a hexagon with side of 50 meters. The initial conditions are the final positions from the previous octahedron formation, which are as follows: Sat1 (4.9, 7.7, -28.8), Sat2 (-22.9, 18.2, -0.1), Sat3 (-35.0, 31.0, -5.1), Sat4 $(-38.1,22.5,-54.8)$, Sat5 (-26.0, -30.2, -53.7) and Sat6 (-66.0, -6.5, -28.1). The same $b$ matrix is used. The new $c$ matrix is:

$$
c=\left[\begin{array}{cccccc}
0 & 800 & 800 & 800 & 800 & 800 \\
800 & 0 & 800 & 800 & 800 & 800 \\
1100 & 800 & 0 & 1100 & 800 & 800 \\
1100 & 900 & 1100 & 0 & 1100 & 800 \\
1100 & 1100 & 900 & 800 & 0 & 1100 \\
800 & 800 & 800 & 800 & 800 & 0
\end{array}\right]
$$

Fig. 6 shows the plot of the maximum eigenvalue of $\mathbf{A}$ matrix for these APF variables when the inter-satellite distance is varied from 1 to 2000 meters. The random external disturbance acting on the formation has a maximum bound of $\pm 10^{-4} \mathrm{~N}$. The simulation was executed for $4 \times 10^{5}$ seconds.

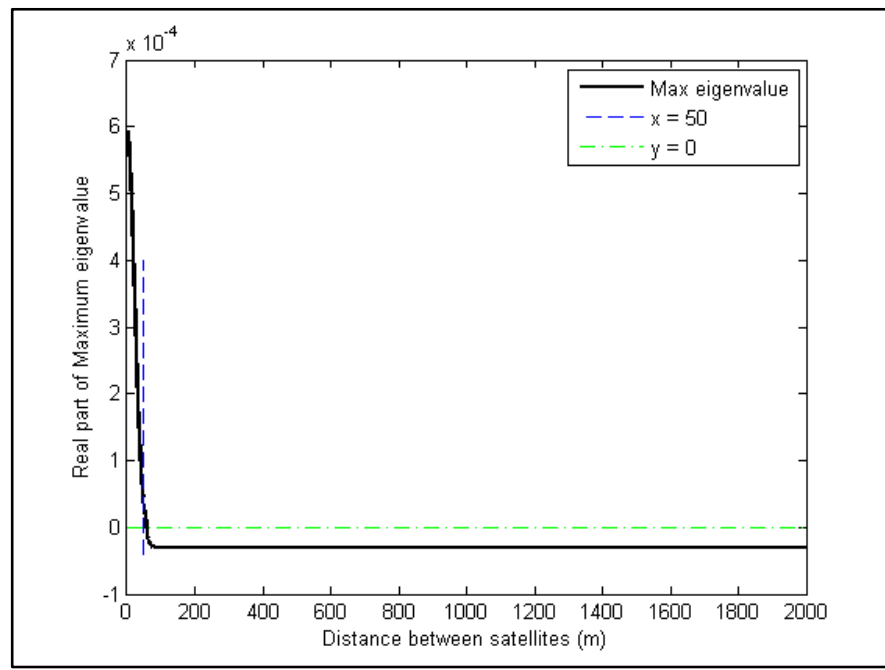

Figure 6: Reconfiguration: Maximum Eigenvalue of the $A$ Matrix

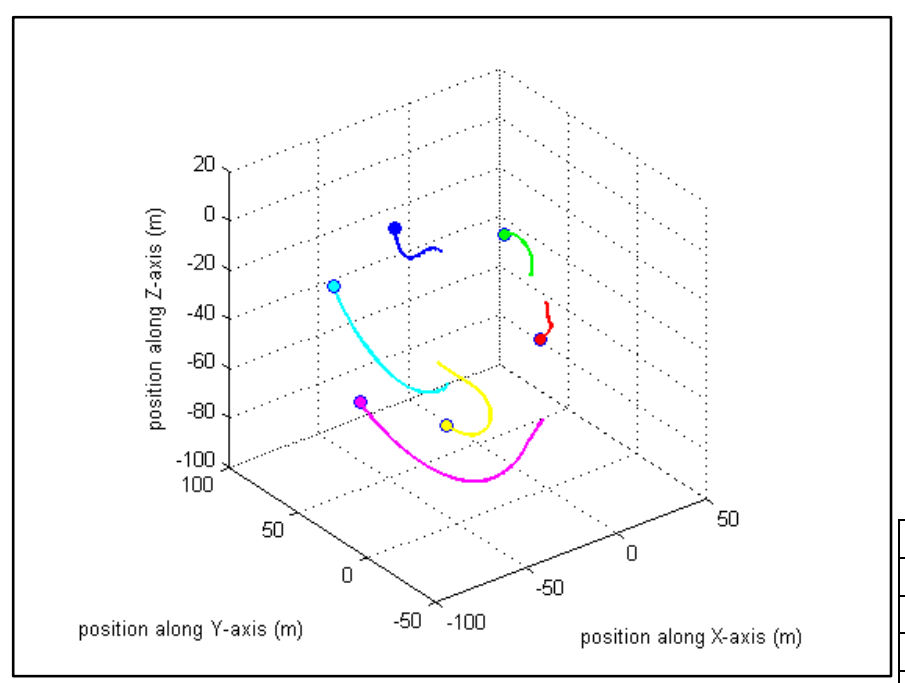

Figure 7: Reconfiguration: Aggregation of Satellites
Fig. 7 shows the trajectory of the reconfiguration from octahedron to hexagonal formation and Fig. 8 shows the final position of the square formation. Fig. 9(a) shows the intersatellite distances and Fig. 9(b) shows the relative velocity between the satellites. The six satellites and the CM are seen to drift while the formation is being formed. Fig. 10(a) shows the norm of the sliding surface and Fig. 10(b) shows the plot of the total magnitude of the force required by the individual satellites for the formation to be achieved. The final intersatellite separation based on the simulations is shown in Table 2 . The simulation results presented in this paper clearly proves the efficacy of the proposed path planning and robust control strategies for small satellite formation flying missions.

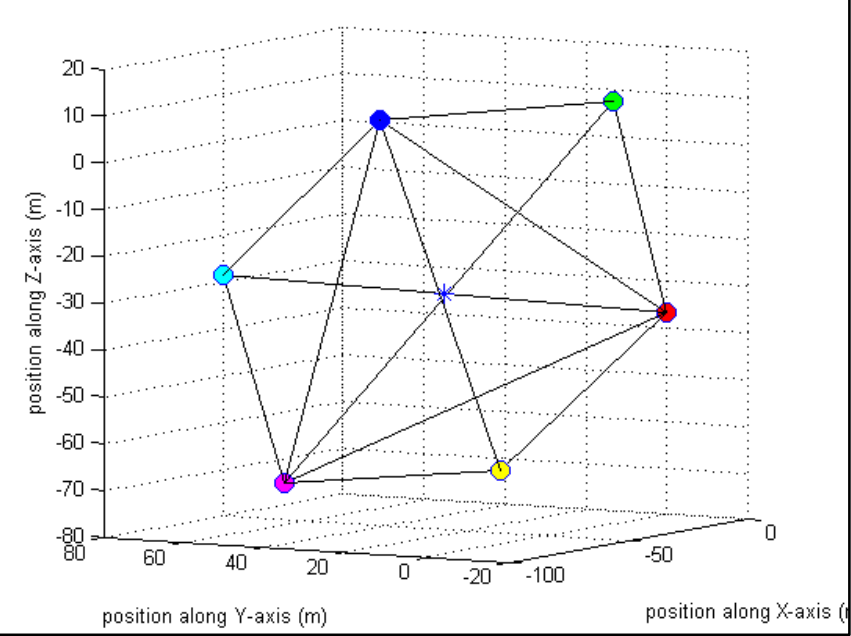

Figure 8: Reconfiguration: Final Formation with the Centre of Mass
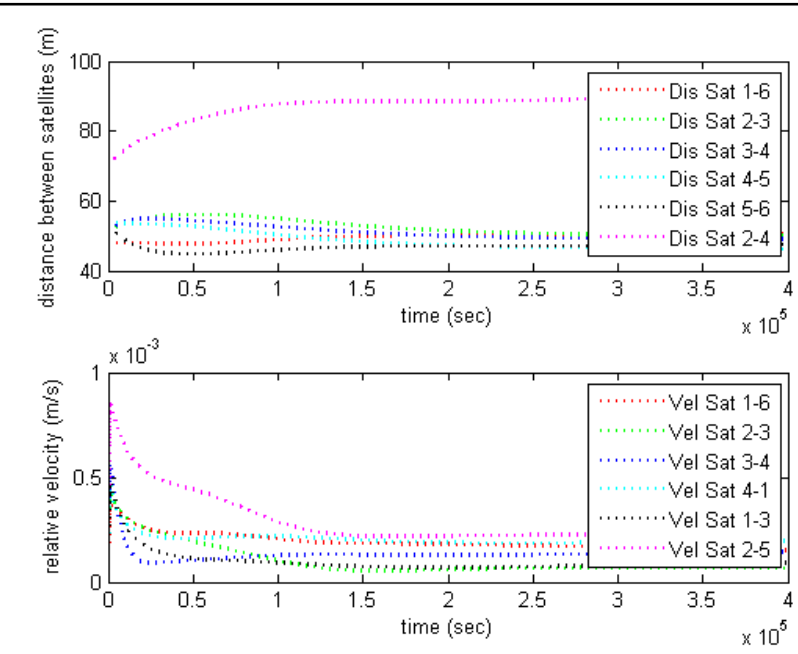

Figure 9: Reconfiguration (a) Distance between the satellites (b) Relative Velocities between satellites

Table 2. Reconfiguration: Final Inter-Satellite Separation

\begin{tabular}{|l|r|r|r|r|r|r|}
\hline & \multicolumn{1}{|c|}{ Sat 1 } & \multicolumn{1}{|c|}{ Sat 2 } & \multicolumn{1}{|c|}{ Sat 3 } & \multicolumn{1}{|c|}{ Sat 4 } & \multicolumn{1}{c|}{ Sat 5 } & \multicolumn{1}{c|}{ Sat 6 } \\
\hline Sat 1 & 0.0 & 50.4 & 77.4 & 94.9 & 89.2 & 52.3 \\
\hline Sat 2 & 50.4 & 0.0 & 50.0 & 91.2 & 108.0 & 82.4 \\
\hline Sat 3 & 77.4 & 50.0 & 0.0 & 49.1 & 82.0 & 79.3 \\
\hline Sat 4 & 94.9 & 91.2 & 49.1 & 0.0 & 46.2 & 72.4 \\
\hline
\end{tabular}




\begin{tabular}{|l|r|r|r|r|r|r|}
\hline Sat 5 & 89.2 & 108.0 & 82.0 & 46.2 & 0.0 & 47.4 \\
\hline Sat 6 & 52.3 & 82.4 & 79.3 & 72.4 & 47.4 & 0.0 \\
\hline
\end{tabular}

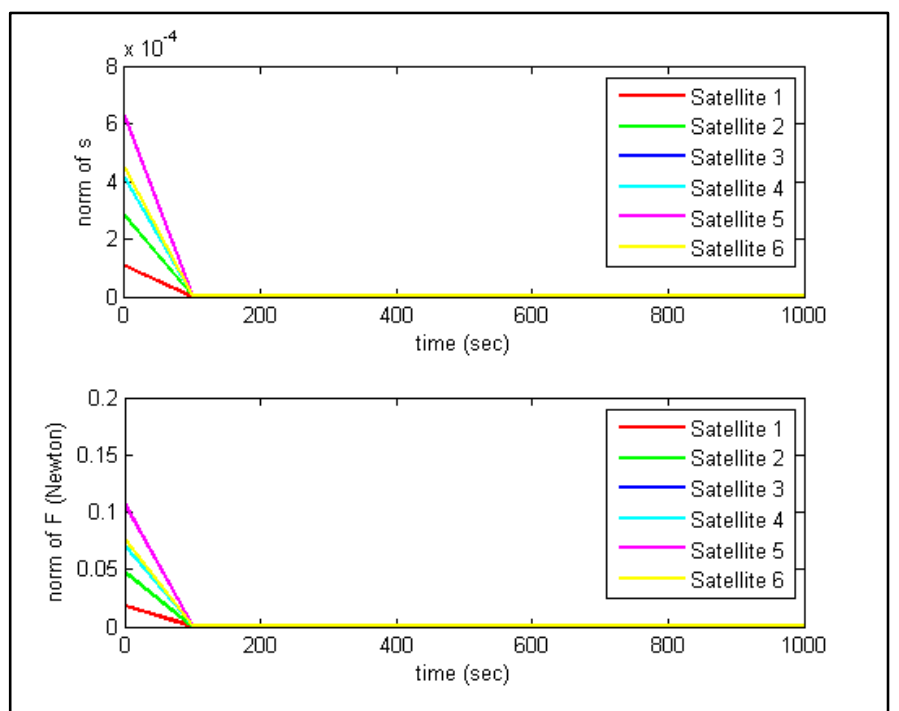

Figure 10: Reconfiguration: (a) Norm of the sliding surface $s_{i}$ (b) Norm of the Force applied by each satellite

\section{CONCLUSIONS}

This paper presents a new method for stability analysis of satellite formations and reconfigurations in Deep Space Environment. The results presented in the paper prove the stability of the formation once the system trajectories hit the respective sliding surface and converge to form the desired geometry. The stability analysis presented in this paper is different from the conventional definition of stability in which, the origin of the state space is the stable point. However, this analysis shows that the stable configuration is represented by the desired final geometry. Moreover, it is shown that the artificial potential field based sliding mode controller results in robust performance in the presence of external disturbance in Deep Space. Simulation results prove the efficacy of the proposed approach for formation and reconfiguration missions using six satellites for forming an octahedron and then reconfiguring to a hexagonal formation. This method of path planning and controller design is computationally less expensive and various formation scenarios using any number of satellites can be achieved by appropriate tuning of the APF and controller parameters. It is envisaged that this concept can be extended to small satellite swarms in future. In this study, continuous-time model has been used, hence further research is needed for developing new control algorithms using discrete-time model for practical applications.

\section{REFERENCES}

[1] A. Das, R. Cobb and M. Stallard, "TechSat 21 - A revolutionary concept in distributed space based sensing," Proceedings of the Guidance, Navigation and Control Conference, AIAA-98-5255, Boston, MA, pp. 1-6, 1998.

[2] C. M. Breder, "Equations descriptive of fish schools and other animal aggregations,” Ecology, Vol. 35 No. 3, pp. 361-370, 1954.

[3] K. Warburton, J. Lazarus, "Tendency-distancemodels of social cohesion in animal groups," Journal of Thoeritical Biology, Vol. 150, pp. 473-488, 1991.
[4] A. Okubo, "Dynamical aspects of animal grouping: swarms, schools, flocks, and herds," Advances in Biophysics, Vol. 22, pp. 1-94, 1986.

[5] V. Gazi, Kevin M. Passino, "A class of Attraction/Replusion Functions for Stable Swarm Aggregations," International Journal of Control, Vol. 77 No. 18, pp. 1567-1579, 2004.

[6] O. Khatib, "Real-Time Obstacle Avoidance for Manipulator and Mobile Robots," The International Journal of Robotics Research, Vol. 5 No. 1, pp. 90-98, 1986.

[7] C. M. Saaj, V. Lappas, D. Richie, H. Schuab and V. Gazi, "Satellite Formation Flying: Robust Algorithms for Propulsion, Path Planning and Control", Proceedings European Control Conference, Budapest, Hungary, 23-26 August 2009.

[8] Gazi, V., "Swarm Aggregations using Artificial Potentials and Sliding Mode Control," IEEE Transactions on Robotics, Vol. 21 No. 6, pp. 1208-1214, 2005.

[9] Gazi, V., Passino, K. M., "Stability Analysis of Swarms," IEEE Transactions on Automatic Control, Vol. 48 No. 4, 2003.

[10] Tang, C. Y., Misawa, E. A., "Discrete Variable Structure Control for Linear Multivariable Systems: The State Feedback Case," Proceedings of the American Control Conference, Philadelphia, Pennsylvania, 1998.

[11] Bandyopadhyay S., C. M. Saaj, Bandyopadhyay B., "Development of Sliding Mode Controller for Small Satellite in Planetary Orbital Environment Formation Flying Missions", International Astronautical Congress, Paper ID: IAC-10-C1.5.6, Prague, 2010 organized by International Astronautical Federation.

[12] Palmer, P., "Optimal Relocation of Satellites Flying in Near-CircularOrbit Formations," Journal of Guidance, Control, and Dynamics, Vol. 29, No. 3, May-June 2006. 